\title{
Patología general de la evaluación educativa
}

\author{
MIGUEL ANGEL SANTOS \\ Universidad de Málaga
}

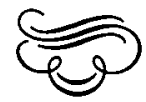

Resumen

El autor plantea en este articulo los problemas que entrañan los procesos de la evaluación institucional educativa en sus diversos niveles (evaluación del alumno, de los Centros, de los programas y Reformas...) y desde diferentes perspectivas (ideológica, política, ética, didäctica, técnica...). Analiza diversas disfunciones y desenfoques de la evaluación escolar, tanto por atrofia como por hipertrofia de algunos de sus elementos, como por la desvirtuación de su práctica y de su significado.

\section{General Pathology of Educational Evaluation}

\section{Abstract}

This issue is concermed with problems derived from the process of Educative Institutional Evaluation at different levels (pupils, Centres, programs and curricula reforms) and different perspectives (ideological, political, ethical, institutional, technical...). The author analizes some educational evaluation disfunctions and missconception due as to the atrophy/hypertrophy of some of its elements as well as to the inappropiate practice and meaning use.

Direcciōn del autor: Travesía Marcos Zapata, 5, ático. 29017 Málaga, España. 


\section{4}

El desarrollo curricular, en cualquiera de sus niveles, está recorrido por un proceso evaluador de triple naturaleza: la evaluación diagnóstica, la evaluación procesual y la evaluación de término. En el buen entendido de que el proceso es circular, no meramente lineal, dinámico en su entraña.

La toma de decisiones, inicial o de proceso, nace de una valoración precisa y de un análisis del planteamiento, de la acción, del contexto y condiciones de la misma y -en su caso- de los resultados.

En el complejo mundo de la educación, por tantos motivos particular y cambiante, se realizan diversos procesos evaluadores del curriculum que están afectados de las patologías más diversas.

Cada uno de los desórdenes que afectan a ese proceso (en el marco de referencia macrocurricular = sistema, mesocurricular = centro y microcurricular = aula) puede ser estudiado en sus signos, en sus síntomas y en su fisiopatología, es decir, en el mecanismo por el que se produce esa «enfermedad».

Hablamos de patología general porque nos interesa el estudio global que siente las bases de cualquier posterior subdivisión analítica. Podríamos hablar también, sensu strictu, de patología social (término introducido por la escuela organicista) de la evaluación, ya que se trata de desórdenes de procesos que afec$\tan$ a colectivos y a relaciones entre individuos.

La evaluación (tanto la de carácter funcional como la investigadora) puede ser manejada para servir los intereses del evaluador ya que éste puede llamar «evaluación» a la operación que desee, puede evaluar aquello que le interese, en las formas y momentos que determine, con los instrumentos que considere oportunos y - desde luego- para utilizarla en los fines que su particular interpretación aconseje.

El poder que dimana del proceso evaluador es tan grande que permite atribuir causalidades arbitrariamente, catalogar éticamente, justificar decisiones y clasificar realidades...

La apariencia de rigor se convierte en una amenaza de perfiles contundentes. Los ribetes científicos disminuyen la capacidad de crítica y prestan un peligroso aval. Si entendemos/practicamos la evaluación como un proceso difícilmente contestable en su concepción, en su desarrollo, en su utilización, difícilmente podrán ser discutidas las conclusiones: «Este alumno ha suspendido», «este centro escolar es malo», «esta experiencia educativa es excelente», «esta actividad tiene que desaparecer», «estas personas tienen que ser excluidas»...

Los criterios que se aplican para la evaluación no siempre se ajustan a patrones rigurosamente elaborados. Y así, una reforma puede considerarse «buena» porque aumenta el número de los conocimientos de los alumnos, un centro puede etiquetarse como «estupendo» porque aprueba la selectividad un porcentaje alto de alumnos presentados y un alumno es considerado «excelente» porque ha contestado correctamente una prueba objetiva.

Más aun, de una evaluación superficial pueden nacer explicaciones explícitas/implícitas que consagran una determinada realidad, estableciendo nexos causales gratuitos. «Este alumno suspende porque no estudia», «ese programa de renovación no funciona porque los profesores son incompetentes», «ese centro tiene eficacia educativa porque posee grandes instalaciones»...

La patología que afecta a la evaluación afecta a todas y cada una de sus vertientes: por qué se evalúa (y para qué), quién evalúa, cómo se evalúa, para quién se evalúa, cuándo se evalúa, qué se evalúa, a quién se evalúa; con qué criterios se evalúa, cómo se evalúa la misma evaluación, etc. 
Algunas patologías lo son en cuanto hipertrofian un aspecto o dimensión que, planteado en su justa medida, sería positivo. Valorar los conocimientos, por ejemplo, no es un error, pero sí lo puede ser el valorar exclusivamente, obsesivamente, los conocimientos, sin tener en cuenta su naturaleza, su importancia, su interés, su adecuación, su coordinación con otros conocimientos, etc.

Otras patologías se deben a la atrofia de funciones que son consustanciales con un estado de salud educativa. No se desarrolla, por ejemplo, un tipo de evaluación democrática (Stenhouse, Elliot, McDonald) en la que tanto el proceso de evaluación como el manejo de la información resultante es responsabilidad directa de los protagonistas de la experiencia educativa.

Hay también carencias, disfunciones... La gravedad de la anomalía dependerá, en parte, de su misma naturaleza y, desde luego, de su intensidad.

\section{SOLO SE EVALUA AL ALUMNO}

En este sentido sí es protagonista el alumno. Se le examina siguiendo una temporalización determinada. Se le dan los resultados, prácticamente inapelables y, en general, se le considera único responsable de los mismos.

No parece concebirse el curriculum sin la evaluación del alumno, pero sí sin la evaluación genérica del mismo. Los argumentos - cargados de lógica- que se utilizan para avalar la ineludible necesidad de la evaluación del alumno no se aplican a otros elementos del curriculum.

A cada alumno se le asigna en el expediente un valor numérico (al menos, cuantificado) que parece ser de su exclusiva responsabilidad. La calificación del alumno - para muchos padres, profesores y para los mismos alumnos- es el resultado de su capacidad y de su falta o derroche de esfuerzos. En el caso de fracasar será el quien deberá pagar las «consecuencias». Sólo él deberá cambiar. Lo demás, podrá seguir como estaba. La evaluación se convierte así en un proceso conservador.

La distinción socorrida de evaluación suficiente y evaluación satisfactoria viene a poner de manifiesto otro flanco de la patología. Porque se utiliza como criterio referencial fáctico la consecución de unos conocimientos mínimos (será el profesor quien lo compruebe según sus particulares criterios), estableciendo en la gama de puntuaciones una comparación con el resto de los escolares a todas luces discutible.

¿Qué es lo que se ha comparado para colocar a los individuos en la escalera de suspensos/aprobados/notables/sobresalientes/matrículas...? No se sabe si se comparan las capacidades de los sujetos, los esfuerzos realizados, los conocimientos adquiridos..., o la suerte de que han gozado. Lo cierto es que la etiquetación que nace de la evaluación educativa figura estampada en impresos, debidamente rubricada por la autoridad académica y refrendada por los sellos oficiales.

Este ejercicio enmascara una injusticia grande, no sólo por la arbitrariedad de asignación, por el capricho atributivo (agravado por la apariencia de rigor científico), sino por la desigualdad radical de condiciones naturales y contextuales. Partir de situaciones desiguales y pretender comparar los resultados utilizando los mismos raseros es una forma radicalmente injusta de ejercitar una aparente justicia.

Lo cierto es que, en este mecanismo, sólo se incluye al alumno, quiera o no, quedando muchos otros responsables del proceso educativo sin esa consideración evaluadora y sin las consecuencias que llevaría aparejadas. 


\section{SE EVALUAN SOLAMENTE LOS RESULTADOS}

Los resultados han de ser tenidos en cuenta dentro del proceso evaluador. Pero no solamente los resultados. Los presupuestos de los que se parte, las condiciones que se tienen, las estrategias que se ponen en marcha, los procesos que se desencadenan, los ritmos de consecución, la proporción rendimiento/esfuerzo..., son también elementos que deben evaluarse. No sólo porque la consecución/no consecución de unos resultados (y el grado de su logro) está supeditada a aquellos factores sino porque ellos mismos constituyen el objetivo de la mirada evaluadora.

En definitiva, no sólo importa qué es lo que se ha conseguido, sino el cómo, a qué precio, con qué ritmo, con qué medios, con cuántos esfuerzos, a qué costa, para qué fines...

Analizar sólo los resultados obtenidos es, cuando menos, parcial. Y la parcialidad suele ir acompañada de imprecisión y de tergiversaciones.

\section{SE EVALUAN SOLO LOS CONOCIMIENTOS}

El proceso de enseñanza/aprendizaje se realiza sobre un cuerpo de conocimientos más o menos estructurados, más o menos interesantes, más o menos conexionados con la práctica, más o menos «autónomos» (los grados de libertad del curriculum pueden ser variables). No se puede aprender en el vacío. Cuando hablamos de «aprender a aprenden - dejando al margen los conocimientosestamos haciendo meras piruetas mentales.

Aprender a aprender es un slogan tan utilizado como desprovisto de sentido real. Porque sólo se aprende aprendiendo.

No se puede, pues, rechazar el aprendizaje de contenidos. Porque son necesarios para articular el pensamiento, para adaptarse a la realidad y para poder manejarla.

Otra cosa es la selección de los contenidos, su articulación, su significación de organizadores del pensamiento.

Ahora bien, limitarse a la evaluación de conocimientos supone un reduccionismo escandaloso. Existen otra serie de pretendidos logros (véase cualquier formulación curricular, véase incluso la legislación de cualquier rango que defina objetivos educativos del sistema) que no se contemplan debidamente en el proceso evaluador: actitudes, destrezas, bábitos, valores...

Una persona que adquiriese un abundante caudal de conocimientos para mejor destruir/oprimir a los otros, un alumno que llenase su cabeza de conocimientos pero que odiase la sabiduría, un individuo con un gran almacén de datos en la cabeza pero incapaz de comunicarse..., no estaría auténticamente formado.

Luego veremos que la evaluación de estas otras facetas no es tarea fácil. Muchos profesores no tienen conciencia profesional de que se trata de aspectos educativos relevantes. Otros no conocen las formas de acercarse a una evaluación adecuada de los mismos. La Administración, conocedora de esas limitaciones, nacidas de una deficiente formación del profesorado, desconfiando de que puedan realizar espontáneamente estas tareas, pretende implantarlas a golpe de «B.O.E.» Es más barato que una larga y concienzuda formación. Pero es totalmente ineficaz. ¿De qué sirvió implantar la evaluación continua cuando los pro- 


\section{7}

fesores seguían instalados en sus viejas prácticas evaluadoras? Sencillamente, para repetir continuamente aquellas prácticas de evaluación memorística.

\section{SOLO SE EVALUAN LOS RESULTADOS DIRECTOS, PRETENDIDOS}

La puesta en marcha de muchos proyectos curriculares que siguen un criterio de encadenamiento lineal (objetivos propuestos-contenidos-métodos-evaluación de objetivos propuestos) no tiene en cuenta la evaluación de aquellos efectos laterales, secundarios, imprevistos.

Estos efectos existen, casi inevitablemente. Y, en ocasiones son mucho más importantes que los resultados buscados directamente en el proyecto.

La pretensión de que el alumno adquiera un elevado nivel de conocimientos, ejercida de manera despótica, puede engendrar una aversión hacia el estudio muy perjudicial.

La pretensión, en algunos centros religiosos, de que los alumnos adquiriesen una práctica religiosa acendrada, llevó a muchos a una postura de rechazo y de resentimiento hacia lo religioso.

Lo mismo habría de decirse de un centro o de una reforma. El resultado directo del programa es evaluado, pero sin tener en cuenta los efectos secundarios (llamémosles así, aunque en ocasiones tengan mayor relevancia que los buscados) que se han generado.

Introducir una mejora en un centro puede suponer, quizás, el enfrentamiento entre dos sectores del claustro o el enrarecimiento de las relaciones entre la dirección y los profesores.

Bien es cierto que los efectos secundarios, en un momento incontrolables e impredecibles, no deben frenar la innovación o la implantación de un programa seriamente estudiado. Lo que decimos es que la evaluación del mismo debe tener en cuenta tanto los resultados que se buscaban como los que se hayan provocado a lo largo del desarrollo curricular.

\section{SOLO SE EVALUAN LOS EFECTOS OBSERVABLES}

Existen efectos que no son directamente observables. Estos efectos - buscados o no- suelen pasar inadvertidos a los ojos del evaluador.

Los modelos de evaluación basados en la programación por objetivos operativos (Mager, 1972), (Bloom, 1972), (Popham, 1975), exigen un modelo correlativo de evaluación que descanse sobre la aplicación de instrumentos de com. probación de carácter experimental.

Se podría objetar que no es posible evaluar los efectos no observables. No es así. Lo no observable no es equivalente a lo no existente. $\mathrm{Ni}$ a lo no relevante. $\mathrm{Ni}$, por supuesto, a lo no evaluable. Al menos, desde una concepción del proceso evaluador que pretenda profundizar en la entraña educativa.

Eisner (1981) dice que la mayoría de los aprendizajes que realiza el alumno en la escuela no se hallan programados en el curriculum explícito.

Las limitaciones metodológicas que impone una concepción experimentalista de la evaluación, no debe hacernos olvidar todo el bloque del iceberg curricular que permanece por debajo de la superficie de las aguas.

Es precisamente un modelo de evaluación más exigente, más riguroso, más ambicioso el que, paradójicamente, nos lleva a explorar otras capas que no son reconocibles «a simple vista» o a golpe de mediciones pretendidamente objetivas. 


\section{8}

Estas dimensiones de la evaluación exigen, eso sí, la utilización de técnicas de exploración adecuadas al intento de llegar a descubrir e interpretar lo oculto del curriculum y de sus resultados.

\section{SE EVALUA PRINCIPALMENTE LA VERTIENTE NEGATIVA}

En la práctica habitual del docente la evaluación está marcada por las correcciones. El mismo lenguaje descubre la actitud predominante: «corregir» significa kenmendar lo errados. El subrayado de las faltas de ortografía es mucho más frecuente que la explícita valoración de las palabras bien escritas.

Sirva esta anécdota de punto de referencia para referimos a esa actitud no solamente mala por negativista sino por lo parcializante.

Una evaluación rigurosa requiere un tramiento holístico de los fenómenos y de los productos. La comprensión de un proceso adquiere sentido en un análisis estructurado y estructurante en el que la interconexión de todos los elementos permite la explicación y el significado.

El desequilibrio de perspectiva hace que la escuela esté más atenta a los errores que a los aciertos de los alumnos y que los equipos de evaluación externa se apresten más a describir problemas y deficiencias que a resaltar valores y logros.

Además de factores actitudinales puede influir en esta dinámica selectiva la mayor facilidad que existe de describir la tensión que la calma, el error que el acierto, la guerra que la paz...

\section{SOLO SE EVALUA A LAS PERSONAS}

Es un error «someter» a los alumnos o a los profesores de un Centro o a los coordinadores de una reforma a una evaluación conclusiva (lo cual encierra juicios de valoración, no meras descripciones de actuación) sin tener en cuenta las condiciones, los medios, los tiempos, los contextos en que se mueven.

Cronbach (1963) distingue tres grandes áreas sobre las que la evaluación toma decisiones: el material de instrucción, los individuos y la regulación administrativa. No son sólo los individuos los responsables de un proceso o de un resultado. Hay que contemplar cuáles son los medios con los que cuentan, las condiciones en las que trabajan y los márgenes de autonomía real con que cuentan.

Los mecanismos de atribución pueden hacer descansar en el sistema, en la carencia de medios, en la presión de los programas oficiales, en la mala organización de los centróos, en la irracionalidad de los calendarios..., toda la responsabilidad de un proceso educativo.

Esos condicionantes/estimulantes existen. Hay que tenerlos en cuenta a la hora de la evaluación. No solamente como coadyuvantes del intento educativo, sino como elementos que pueden ser modificados y/o mejorados para un posterior desarrollo.

\section{SE EVALUAN DESCONTEXTUALIZADAMENTE}

Bertalanffy $(1976,1978)$ plantea la necesidad de tener en cuenta un contexto amplio cuando se pretende comprender la realidad de un sistema actuante.

Pretender dar significado a la actuación de un alumno desde la óptica y el código del evaluador, prescindiendo de las claves de interpretación del contexto, es vaciar de contenido la realidad (Oates, 1975). 
Encasillar un Centro dentro de la plantilla elaborada por el evaluador con unos criterios genéricos de pretendida validez, es negarse a entender todo lo que sucede en el mismo.

Una calificación sobresaliente puede ser considerada «algo despreciable» en un contexto determinado. Una clase indisciplinada puede estar en el eje de la admiración de la mayoría de los alumnos de un Centro. Una experiencia pedagógica «modélica puede ser valorada en su contexto de forma negativa...

Si el evaluador, en aras de una pretendida objetividad, busca la creación y realiza la aplicación de instrumentos de medida fiables y válidos técnicamente y no tiene en cuenta la realidad viva, compleja y dinámica de ese todo, de ese sistema organizativo que tiene en sí mismo los códigos sintácticos y semánticos, se verá enredado en un caudal de datos muertos y desprovistos de auténtico significado.

La actuación de un alumno podrá ser atendida e interpretada justamente (con justeza y con justicia) en el marco de innumerables redes codificadoras que se producen en el sistema del aula que, a su vez, está en conexión con el sistema del Centro que, a su vez...

«La optimización de un sistema es posible, siempre y cuando nos acerquemos a las peculiaridades específicas de la estructura y comportamiento de cada sistema, y en función precisamente de ese conocimiento específico» (Pérez Gómez, 1985).

\section{SE EVALUA CUANTITATIVAMENTE}

La pretensión de atribuir números a realidades complejas es un fenómeno cargado de trampas en el área de la educación.

En las calificaciones escolares utilizamos escalas nominales, ordinales y de razón. Un aprobado es distinto de un suspenso, un 5 es una nota inferior a un 8 , y un 6 es el doble de un 3. Parece que todo está claro, que todo es muy preciso.

El peligro de la evaluación cuantitativa no es solamente la imprecisión sino - y sobre todo- la apariencia de rigor. «La asignación de números de una manera mecánica, como es común en los procedimientos cuantitativos, no garantiza la objetividad». (Cook, 1986).

Pero, como aparentemente tiene objetividad, genera en los usuarios y destinatarios una tranquilidad mayor que mata las preguntas más hondas.

Algunos padres de alumnos de EGB que ahora reciben informes sobre la marcha de sus hijos con la expresión «Progresa adecuadamente», preguntan a sus profesores: «Progresa adecuadamente, ¿equivale a un 8 de antes?». La puntuación parece un lenguaje más claro. Engañosamente más claro. El padre tiene, además, el punto de referencia de otros alumnos que tienen puntuaciones de 4,669 .

También el profesor puede sentirse más aseguro» después de estampar sobre la prueba objetiva, corregida con una fórmula matemática $(P=A-E / N-1)$, el número exacto, con decimales, correspondiente a la calificación: Además, puede establecer claramente, matemáticamente, la línea divisoria del apto/no apto.

El alumno, con este procedimiento calificador, sabe lo que tiene que estudiar, cómo ha de estudiarlo y - después de la calificación- sabe cuánto ha aprendido.

El problema, además del que entraña la pretendida objetividad de esas pun- 


\section{0}

tuaciones, estriba en que la luz cegadora de esa «claridad» no deja ver cuestiones más importantes:

- ¿Cómo aprende el alumno?

- ¿Cómo relaciona lo aprendido?

- ¿Cómo inserta los nuevos conocimientos en los ya asimilados?

- ¿Para qué le sirve lo aprendido?

- ¿Ha disfrutado aprendiendo?

- ¿Estudiaría «esas cosas» por su cuenta?

- ¿Tiene ganas de aprender cuando terminan las pruebas?

- Etcétera.

\section{SE UTILIZAN INSTRUMENTOS INADECUADOS}

Es una reciente recopilación de instrumentos en curso para la evaluación de centros escolares (Plaza, 1986), se ha podido comprobar que la casi totalidad tiene una configuración estática, anecdótica, aséptica, cuantificadora, descontextualizada, etc.

Los cuestionarios, escalas de estimación, listas de control, plantean el interrogante de la triple adecuación realidad-percepción, percepción-interpretación, interpretación-expresión. Se añade a esta problemática la complejidad de la explicación de los datos cuantificados. Valorar con 3 puntos sobre 10 el funcionamiento de la biblioteca del Centro nos deja en la duda de si la baja puntuación se debe a algunas de estas causas (o a otras posibles):

- No hay libros

- No está actualizada la documentación

- No existen ficheros que permitan la localización rápida y fácil

- No existen locales

- No hay tiempo para su apertura

- No existe personal que la atienda

- No hay afición por la lectura

- Los libros no tienen interés para los alumnos

- No están informados los alumnos de sus normas de funcionamiento

- Existe mucho ruido y es difícil la concentración

- El bibliotecario es una persona indeseable

- Está mal visto entre los alumnos acudir a la biblioteca,

- Etc.

Decir que el $48 \%$ de los profesores valoran con una media de 3 puntos el funcionamiento de la biblioteca, es un modo de esconder, bajo la cifra porcentual, las innumerables causas que han llevado a los opinantes a conceder una puntuación.

Ese modo de evaluar hace difícil la comprensión de la realidad, su interpretación y, obviamente, la posibilidad de efectuar mejoras que solucionen los problemas o potencien los aciertos.

Lo mismo sucede en el aula. Un instrumento de valoración «objetivo» no puede estar más cargado de subjetividad/arbitrariedad. He aquí una escala de ambigüedad que no siempre es tenida en cuenta:

- ¿Lo que aparece en el curriculum como contenido mínimo es lo realmente importante, valioso, interesante...?

- ¿Lo que ha seleccionado el profesor para la prueba, es significativo de lo que tenía que aprender? 
- ¿Lo que pregunta el profesor es exactamente lo que quiere saber sobre lo que ha aprendido el alumno?

- ¿Lo que el alumno lee es lo que el profesor ha querido preguntar?

- ¿Lo que responde es exactamente aquello que sabe sobre la cuestión?

- ¿Lo que interpreta el profesor es lo que el alumno realmente ha expresado?

- ¿La valoración corresponde a lo que el profesor entiende que ha expresado el alumno...?

Nada digamos de la dificultad de mejorar el aprendizaje a través de ese tipo de pruebas. A lo sumo, el alumno prestará atención al mejor modo de contestarlas en próximas ocasiones. Lo cual es distinto al propio proceso de enseñanza/aprendizaje.

Existe otra complicada cuestión en la aplicación habitual de este tipo de pruebas «objetivas» bajo el pretexto de que son más justas, ya que «miden» a todos por igual. Lo cierto es que no existe mayor arbitrariedad que la de querer amedir» de la misma forma a personas que son radicalmente diferentes. Así, un alumno tímido no deseará realizar una entrevista o examen oral. Quien no se expresa bien por escrito, preferirá una prueba...

La naturaleza de la prueba lleva dentro de sí un componente sesgado de valoración, independientemente del tipo de contenidos que plantee y de su forma de presentarlos.

\section{SE EVALUA DE FORMA INCOHERENTE CON EL PROCESO DE ENSEÑANZA/APRENDIZAJE}

El camino es circular, no rectilíneo y unidireccional. Se evalúa como se enseña y se enseña como se evalúa. O más bien, se estudia para la evaluación. De tal forma que es este proceso el que dirige el aprendizaje. Los alumnos estudian para el examen. No sólo en función de ese momento sino «de formas que les permita hacer frente al mismo con suficientes garantías de éxito.

Si el examen consiste en una prueba objetiva de Verdadero-Falso, estudiará de forma distinta que si se le anuncia una prueba de ensayo o si se le exige un diseño creativo. Es más, los alumnos tratarán de acomodarse a las expectativas del profesor, a sus códigos de valor.

La incoherencia se establece cuando se quiere realizar un aprendizaje por comprensión y se realiza luego una prueba de carácter memorístico, rígido y repetitivo.

Un proceso de enseñanza basado en la explicación oral, se cierra con un modelo de examen escrito.

Un modo de trabajo asentado sobre el grupo concluye en una evaluación individual.

Un proceso de enseñanza/aprendizaje teóricamente asentado sobre el desarrollo integral del individuo, acaba con una evaluación exclusivamente preocupada por los conocimientos adquiridos.

Otras veces, el enfoque exclusivo en el aprendizaje de contenidos intelectuales pretende concluirse con una evaluación que va mucho más allá de lo que se ha trabajado.

En el área de la didáctica se puede rizar el rizo al exigir el profesor la realización de una prueba objetiva por él diseñada sobre el tema: «Importancia, necesidad y valor del proceso de autoevaluación en la enseñanzas. 


\section{SE EVALUA COMPETITIVAMENTE}

Creo que una de las ventajas que encierra el enfoque cualitativo es el afinar la sensibilidad del evaluador ante los procesos.

El auténtico significado de un proceso educativo se encuentra en el análisis de todos los elementos que lo integran y que lo circunscriben. Pero no en el contraste, comparación y competencia con otros procesos similares, diferentes o antagónicos.

La evaluación habitual del alumno parece cobrar sentido en la comparación y la competencia. Para algunos padres, profesores y alumnos importa el cuánto del aprendizaje. Y que ese cuánto esté por encima de...

El carácter positivo que se pretende dar a la emulación en algunos ámbitos educativos, se trastoca frecuentemente en una burda o camuflada contienda. «Saber más que», «saber menos que», son puntos de referencia cruciales.

Lo mismo sucede con la evaluación de Centros. La valoración cuantitativa es propensa a la comparación. Las escalas tienen en ella su justificación más evidente. Este es un Centro «mejor este es un Centro que ha obenido 90 puntos sobre 100 , se dice.

La localización de la mirada se sitúa en el exterior del proceso mismo, con el agravante de que se establecen comparaciones arbitrarias ya que pocas veces se parte de condiciones, medios, historia, personas y fines similares.

Por otra parte, es frecuente justificar la comparación con el pretexto de que sirva de estímulo y emulación. Durante toda la enseñanza primaria y secundaria tuve que trabajar con émulos. Cada alumno tenía un compañero que era un competidor. Con él había que medirse. El éxito consistía en aventajarle.

Se desvía la atención del auténtico proceso de aprendizaje de cada individuo, de cada aula, de cada Centro. Y si es posible pensar que el «ganador» se sentirá satisfecho y el «perdedor» estimulado, no es menos imaginable que el primero se sienta ridícula y estúpidamente orgulloso y el segundo, humillado.

En las evaluaciones que he visto realizar sobre los profesores (por sus alumnos), siempre he visto solicitar y exigir cautelas para que los resultados no se hiciesen públicos a través de escalas valorativas. «No se trata de establecer comparaciones», se dice. Los profesores no consideran positivo que se publiquen unas listas donde unos aparecen como mejores y otros como peores. Pero colocan, sin ambages, las calificaciones escalonadas de sus alumnos, muy bien matizadas con decimales...

\section{SE EVALUA ESTEREOTTIPADAMENTE}

Los profesores repiten una y otra vez sus esquemas de evaluación. Cada año los alumnos se preocupan de saber cuál es la costumbre evaluadora del profesor.

De forma casi automática, el profesor tepite sus fórmulas. Ni siquiera negocia con los alumnos el planteamiento habitualmente practicado. Al comienzo de curso fija el número, el momento, la forma y los matices.

¿Ha sometido a evaluación sus propios mecanismos de evaluación? No de una forma rigurosa. Cuando ha pensado en ello ha sido para confirmar los estereotipos.

En un curso con cinco asignaturas un alumno deberá «someterse» a cinco proyectos diferentes de evaluación. Los profesores evalúan de formas muy diferen- 
tes. Pero cada profesor, en los diferentes cursos en que imparte docencia, evalúa de una forma idéntica.

Se multiplican los trabajos sobre evaluación, pero no son los protagonistas - los propios profesores- los que emprenden esta labor. Stenhouse (1984) dice que lo que mejora la acción educativa no es tanto la investigación sobre los profesores sino la de los profesores que analizan su propia actividad.

En los Centros, la mecánica de la confección de las Memorias tiende a convertirlas en pura rutina. Y, en la medida que se establezcan pautas ageneralizables», esas rutinas serán similares en casi todos los Centros. Tan similares como inútiles.

\section{NO SE EVALUA ETICAMENTE}

Además de los problemas técnicos acechan al proceso evaluador numerosos conflictos de carácter ético.

La evaluación puede convertirse en un instrumento de opresión. ¿Que sucedería en las aulas si el profesor estuviese desprovisto del «armas de la evaluación? Cuando se articula el proceso de enseñanza/aprendizaje sobre el resultado de la evaluación -más que sobre la riqueza y profundidad del saber- se corre el riesgo de la manipulación y el sometimiento del alumno. La shora de la verdad» no es la del aprendizaje sino la de la evaluación. Cuando es el profesor quien lo decide todo respecto a ese momento decisivo, todo el poder descansa en sus manos.

La evaluación ha sido un instrumento de control, de amenaza e, incluso, de vanganza, respecto a algunos alumnos que se han permitido ejercitar el derecho a la crítica, a la discrepancia o la indisciplina.

Cuando, apoyándose en la evaluación, se establecen nexos causales poco rigurosos, habrá que pensar cuáles son las intenciones y los intereses que motivan la manipulación.

En la evaluación institucional se plantean numerosos problemas de carácter ético. Uno de ellos es la confidencialidad de los informes. ¿De quién son los datos? ¿Para quién son los datos? No puede revelarse el contenido de informes identificables, tanto por lo que respecta a su emisor como al destinatario de los juicios.

La independencia de los equipos que realizan evaluación externa al servicio de la Administración o de entidades que financian los proyectos, no siempre está garantizada. John Elliot, Helen Simon y Savel Kousner insisten (Elliot et alt., 1986) en la necesidad de conseguir la independencia de los técnicos frente a las posibles exigencias del poder (académico, político, financiero, etc).

A nadie se le oculta que una evaluación puede ser dirigida, condicionada o manipulada al servicio de unos intereses determinados, o de unos decisiones pretendidas: retirar subvenciones, modificar proyectos, sustituir personas, clausurar centros, cortar experiencias, etc.

\section{SE EVALUA PARA CONTROLAR}

La evaluación en educación, paradójicamente, no suele ser educativa. No repercute en la mejora del proceso. La evaluación se cierra sobre sí misma, constituye un punto final. 


\section{4}

Se habla de «calificación final». Cuando es justamente un momento más en el momento de mejora. No se debe confundir control con evaluación, aunque las dos funciones pueden ser necesarias. El poder sancionador de la evaluación no constituye su esencia más rica, más dinámica.

Cuando los profesores se niegan a explicar a sus alumnos (o se muestran reticentes a hacerlo, por pensar que se trata de una pérdida de tiempo) de dónde proceden las calificaciones que les han atribuido, están desaprovechando un buen elemento de aprendizaje.

Disparar con los ojos vendados sobre una diana sin saber dónde se ha producido el impacto, hace imposible la mejora de la puntería en ensayos posteriores. Solamente conociendo el resultado se puede mejorar en próximos disparos. Pero, no solamente será preciso conocer y analizar lo que ha sucedido, sino planificar los nuevos procesos en función de aquello que se ha descubierto como fracaso o acierto.

Ese efecto retroalimentador se suele perder en las evaluaciones educativas. Y así, la evaluación que suponen las memorias finales de los Centros, en nada iluminan el nuevo proyecto del curso siguiente. Los puentes de la eficacia están volados por la falta de análisis, la inercia inveterada y la despreocupación de muchos profesionales.

El mundo educativo está lleno de situaciones en las que no se aprovecha esta riqueza potencial que lleva en su interior el proceso evaluador. Los profesores/alumnos repiten los mismos errores casi con obstinación, los cursos que finalizan una especialidad en nada benefician con el análisis de su historia a los cursos siguientes, los Centros no aprenden de la reflexión rigurosa sobre su experiencia...

«La evaluación es el proceso de diseñar, obetener y proporcionar información útil para juzgar alternativas de decisión», dice Stuffelbeam (1971). Según este planteamiento, cuando la información no repercute en la toma de decisiones perdería su misma esencia.

La nueva decisión, al ser puesta en práctica, deberá ser también evaluada. Este proceso dinámico, abierto, facilita el cambio y posibilita la mejora.

\section{SE EVALUA PARA CONSERVAR}

Hemos apuntado ya que la evaluación suele cerrarse sobre sí misma, limitándose a desarrollar una función sancionadora. En ese sentido, no impulsa el cambio.

Pero, hay algo más grave, escondido en la patología de su funcionalidad: La utilización de la misma para justificar el statu quo del profesor, de la dirección del Centro, de la Administración Escolar.

Si los malos resultados o el mal funcionamiento del proceso se pueden atribuir libremente (arbitrariamente) a quien se quiera con la apariencia de la objetividad, del rigor científico y del argumento de autoridad del que maneja la evaluación, el «interesado en no cambiar» que, a la postre, tiene en su poder la interpretación de la evaluación, podrá mantener tranquilamente los mismos planteamientos.

Y los cambios introducidos afectarán a las capas y esferas menos comprometedoras, más superficiales o más económicas a la hora de realizar el diagnóstico de la innovación y la estrategia y la temporalización de la misma. 
Lo cierto es que se evalúa constantemente en la escuela, pero se cambia muy poco.

\section{SE EVALUA UNIDIRECCIONALMENTE}

La evaluación tiene sentido «descendente». El Ministerio «evalúa» a inspectores, los inspectores «evalúan» a los directores escolares, los directores «evalúan» a los profesores, los profesores aevalúan» a los alumnos. Si bien es cierto, que en la parte más baja es donde más se nota el peso de esa función.

No se produce una evaluación es sentido eascendentes. $\mathrm{Ni}$, desde luego, en sentido horizontal.

Un modelo de evaluación democrático supone que los interesados manejan la evaluación. Deciden sobre ella. Y ellos son los que dicen lo que piensan, los que analizan lo que hacen.

«Para conocer la realidad y sus significados relevantes hay que sumergirse en el curso real y vivo de los acontecimientos y conocer las interpretaciones diversas que hacen de las mismas aquellos que las viven» (Pérez Gómez, 1983).

Sólo la evaluación democrática puede propiciar un cambio en profundidad. Esa evaluación, solicitada o emprendida por la comunidad en cuestión, revierte sobre ella en informes rigurosos, claros y significativos.

No es que la evolución democrática pueda prescindir de los expertos en evaluación. Pero no son éstos los que pueden atribuir significado de espaldas al mundo de los protagonistas. $Y$ no son los expertos los que tienen el poder de la evaluación. Los expertos tienen la técnica, pero no las claves de la interpretación y no los resortes del poder pedagógico.

En la evaluación externa sólo la participación directa de los protagonistas podrá dar fiabilidad y validez a la lectura de los signos, a su metalectura posterior y a su utilización para el cambio.

Los que desean conocer el resultado de la evaluación y los que la llevan a cabo son integrantes de un proceso. También por eso la evaluación debe ser de. mocrática (McDonald, 1976).

\section{NO SE EVALUA DESDE FUERA}

Una experiencia educativa necesita la evaluación externa para poder realizar una mejora sustantiva. No hacerlo así significa cerrar el horizonte valorativo y arriesgarse a la miopía y a la deformación óptica del que mira algo que está sobre sus mismos ojos.

Cerrar el círculo de la planificación de objetivos, métodos, evaluación, desde la exclusiva mirada del protagonista del proceso entraña el riesgo de quedarse atrapado en la propia limitación.

El evaluador externo goza de un punto de vista privilegiado, en cuanto tiene:

- Una distancia afectiva de la dinámica y del resultado.

- Unos criterios de independencia respecto al resultado.

- Unos puntos de referencia más amplios y complejos.

- Una mayor disponibilidad en el tiempo y la dedicación.

Esto no quiere decir que se pueda prescindir de los protagonistas de la experiencia, como apuntábamos más arriba. Tanto es así, que dentro de la metodo- 


\section{6}

logía etnográfica de la evaluación es aconsejable la aportación del observador participante.

No decimos que el evaluador externo tenga que ser aséptico o ejercer una neutralidad que ni es posible ni siquiera deseable. El evaluador no solamente mira, sino que busca. Y para ello ha de interpretar. Y la interpretación necesita unos códigos estructurados en los ejes de una sólida teoría.

\section{NO SE HACE AUTOEVALUACION}

La autoevaluación es un proceso de autocrítica que genera unos hábitos enriquecedores de reflexión sobre la propia realidad.

Dice Popper que realizamos más progresos al reflexionar sobre nuestros errores que al descansar en nuestras virtudes.

John Elliot plantea la autoevaluación de los Centros como una tarea de investigación en la acción (1986).

Las técnicas de diseño, de exploración y de interpretación y utilización de esas autoevaluaciones han de ser conocidas, asumidas y llevadas a término por los miembros de la comunidad escolar. ¿Tendría sentido un proyecto de evaluación que no fuese deseado, aceptado y conocido por los protagonistas de la experiencia?

Muchas de las reticencias, de los miedos y de los conflictos que provoca una evaluación externa/impuesta desaparecen, ipso facto en el proceso de autoevaluación.

También los alumnos pueden/deben practicar estos procesos autoevaluadores. El profesor debe poner en sus manos los instrumentos precisos para ello. $\mathrm{Y}$ ha de negociar con él el reparto de las cotas de decisión que lleva consigo la evaluación.

No es aceptable la práctica del juego autoevaluador, en la cual el alumno hace reflexiones y análisis sobre su aprendizaje, pero no puede materializar el resultado en una parcela de las calificaciones.

$\mathrm{El}$ profesor suele actuar con muchas reticencias en este sentido, suponiendo que el alumno no se calificará con criterios justos, por falta de objetividad o por carencia de referencias exteriores que le sirvan de contraste.

Contaré una experiencia reveladora al respecto. En un claustro de profesores de un Centro Escolar oí cómo los profesores se resistían a practicar la autoevaluación con sus alumnos por considerar que eran ademasiado pequeños, que no tenían capacidad para ellos. Ese mismo día, en un Departamento universitario, otros profesores argumentaban que «la autoevaluación de los alumnos no era posible en el ámbito universitario porque no tenían experiencia de haberla practicado en Centros de niveles inferiores».

Cuando los profesores utilizan estas excusas no están en condiciones de realizar esa experiencia. Del mismo modo que cuando dicen que el número de alumnos o las condiciones de trabajo y de tiempo les obligan a realizar una evaluación superficial y masiva, no podemos afirmar con seguridad que con menor número y mejores condiciones harían las cosas de otro modo.

\section{SE EVALUA «DISTEMPORALMENTE»}

Entiéndase aquí el prefijo en su acepción griega (dis $=$ mal), a sabiendas de que la palabra compuesta no debería utilizarse. 
Cuando se habla de aevaluación continuas se quiere decir algo que casi nunca se hace. Más bien, ha tenido el efecto contrario como apuntábamos anteriormente. El deseo de eliminar una mala forma de evaluar (anecdótica, memorística, superficial...) llevó a multiplicar las veces en que esto se hacía.

Porque el núcleo del concepto está más en el cómo que en el cuándo, más en el fondo que en la forma. El concepto exigía una actitud distinta, unos métodos diferentes, un enfoque nuevo. Pero no hacer muchos más controles del mismo tipo que los que se hacían.

No se hace, pues, una buena evaluación sincrónica respecto al proceso de aprendizaje o, más ampliamente, de educación.

Pero tampoco se hace una adecuada evaluación diacrónica. Entendemos por «evaluación diacrónicas aquella que se realiza a través del tiempo, con una perspectiva temporal que ofrezca nuevos elementos de referencia.

Veamos un ejemplo relativo a la evaluación de Centros escolares. ¿Cómo evalúan los ex-alumnos de un Centro su experiencia educativa una vez transcurridos uno, dos, tres, cinco años? El «antiguo alumno» tendrä̌ unos elementos de comprobación/contrastación que no tiene el individuo que está inmerso en la experiencia. Sabe si «aquello» sirvió para algo, si le permitió adaptarse a los problemas...

Además de estas ventajas se encierra la de una independencia más clara, más firme, más abierta. Ahora puede opinar sin ningún tipo de reticencias.

En ese grupo que llamamos ex-alumnos de un Centro habrá que integrar a los que lo abandonaron y a los que - de una u otra forma - se les instó a cambiar de Centro o a quedarse en la calle.

\section{NO SE HACE PARAEVALUACION}

La evaluación a la que aquí nos referimos se ciñe a cuestiones de la misma indole que la evaluación.

Veámoslo con algunos ejemplos. Un evaluador externo analiza la eficacia de una institución militar en la formación de sus alumnos. Y puede llegar a la comprobación de que realmente existe un buen planteamiento en los métodos que intentan lograr esos objetivos. Realmente se han conseguido. Supongamos que esos objetivos «no son deseables», que esos métodos utilizados «no son éticos» y que esos logros «son contraproducentes para la formación de la persona». ¿Termina la función evaluadora con el informe de que el funcionamiento es bueno porque se está desarrollando el programa dẹ forma eficaz?

Un sistema educativo de un país totalitario incluye entre sus objetivos la formación de los profesores y de los alumnos en la filosofía y en el proselitismo sectario. Y el análisis del funcionamiento del sistema, permite al evaluador comprobar que se están consiguiendo esos objetivos de forma rápida, eficaz y profunda. Termina su tarea al realizar un informe en el que se describa y analice la eficacia del sistema?

Entendemos que no. De alguna manera Scriven (1973) se refiere a esta cuestión cuando diferencia la evaluación de la estimación del logro de los objetivos.

Nosotros pensamos que la «paraevaluación» (para = junto a, al lado) supone un análisis de contenidos y un juicio de valor que va más allá de la simple descripción y análisis de la coherencia del programa y de la eficacia del mismo. 


\section{NO SE HACE META-EVALUACION}

El proceso de evaluación es tan complejo que ha de ser necesariamente evaluado para poder atribuirle un valor.

En cualquiera de sus vertientes puede encerrar numerosas trampas, numerosos riesgos, numerosas deficiencias. Por eso se hace imprescindible establecer criterios que permitan evaluar los mecanismos de evaluación.

No solamente para analizar el rigor del proceso, sino para ver cómo esa misma función condiciona lo que se estaba haciendo. Ahora, la evaluación de un programa se convierte en otro elemento que ha de ser evaluado proque está actuando como una variable poderosa que puede distorsionarlo o modificarlo sustancialmente.

En esta vuelta de tornillo, vuelven a aparecer todos los componentes de la evaluación con un nuevo nivel de complejidad: quién metaevalúa, para quién, por qué, cuándo, con qué instrumentos, qué, de qué modo...

Un proceso riguroso de metaevaluación no sólo permitirá valorar de manera rigurosa los resultados, sino que permitirá tomar decisiones eficaces para mejorar el planteamiento, la dinámica y los modelos de evaluación.

\section{Referencias}

Bertalanffy, V. (1976). Teoría general de sistemas, México, F.C.E.

BERTALANFY, V. (1978). Tendencia de la teoría general de sistemas, . Madrid, Alianza.

Bцоом, D. S. (1971). Taxonomía de los objetivos de la educación. Buenos Aires: Ateneo.

COOK. T. D. y REICHARDT. Ch. S. (1986). Métodos cualitativos y cuantitativos en investigación educativa, Madrid, Morata.

Cronbach, L. (1963). Coutse improvement through evaluation, Teachers college Record, 64.

EISNER, E. (1981). The Methodology of qualitative Evaluation: the case of Educational Connoiseurship and Educational Cristicism, Stanford University, Unpublish.

EụorT. J. (1983). aAction-research»: Normas para la autoevaluación en los Colegios, Cambrigde Institute of Education. Multicopiado.

Eu.uoTT, J. (1986). Investigación-acción en el aula. Valencia: Generalitat Valenciana, Consellería de Cultura.

MAGER. R. F. (1974). Formulación operativa de objetivos didácticos. Madrid: Morata.

MCDonalD, B. (1976). Evaluation and the control of Education, en Tawney, D. (Ed.) (1976): Curriculum Evaluation today: trends and Implications, Londres: Mc Millan.

OATES, J. (1975). People in cities: An Ecologial Approach. Open University Press.

Pérez GÓmEZ, A. (1983). La evaluación didáctica. En Gimeno Sacristán, J. y Pérez Gómez A. (1983). La enseñanza, su teoría y su práctica, Madrid: Akal.

PÉrez GOMEZ, A. (1985). La comunicación didáctica. Departamento de Didáctica y Organización, Málaga.

Plaza Del Río, F. (1986). Modelo de evaluación cualitativa de un centro docente. Memoria de licenciatura, Universidad de Málaga.

Popham, J. (1975). Problemas y técnica de evaluación. Madrid: Anaya.

SCRIVEN, M. (1973). Goal free evaluation. En F. R. House School Evaluation. The politics and process. Berkeley: McCut Cheon Publisher.

STENHOUSE, L. (1984). Investigación y desarrollo del curriculum, Madrid: Morata.

STUFEELBEAM, D. L. (1971). Educational evaluation and Decision Making, Bloomington. 


\section{Libros recibidos}

Acción Educativa (Madrid)

VV.AA. Metodología activa. Reflexiones, experiencias.

Barcanova (Barcelona)

BENEDITO, V.: Introducción a la Didáctica. Fundamentación teónica y diseño cumicular.

IMBERNON, F.: La formació permanent del professorat.

CEAC (Barcelona)

SOLÉ. I.: L'ensenyament de la comprensió lectora.

Escuela Española (Madrid)

BARBERÁ, V.: Proyecto educativo, plan anual del centro y programación docente.

CUENCA, F.: Cómo estudiar con eficacia.

FERNÁNDEZ, E.: Teatro español del siglo XX para la infancia y la juventud.

GOMEZ. J.: Trastomos de la adquisición del lenguaje. Valoración y tratamiento.

Eudema (Madrid)

BELTRÁn, J. et el.: Psicología de la educación.

Gedisa (Barcelona)

FRIANT, J., y L'HOSPITALIER, Y.: Juegos lógicos en el mundo de la inteligencia artificial.

LEAL, A.: Construcción de sistemas simbólicos: la lengua escrita como creación.

ORTigues, M. C., y ORTIGUes, E.: Cómo se decide una psicoterapia de niños.

Generalitat de Catalunya (Barcelona)

FORNER, A.: El fenómeno bilingüe.

Graó (Barcelona)

FORNER, A.: La comunicación no verbal. Actividades para la escuela.

Martínez Roca (Barcelona)

SALDAÑA, C., y ROSELL, R.: Obesidad.

Morata - Ministerio de Educación y Ciencia (Madrid)

LOUGHIN, C. L., y SUINA, J. H.: El ambiente de apredizaje: Diseño y organización.

Paidós - Ministerio de Educación y Ciencia (Barcelona y Madrid)

APPLE, M. W.: Educación y poder.

CAMPION, J.: El niño en su contexto.

NICKERSEN, R. S. et al.: Enseñar a pensar.

WOODS, P.: La escuela por dentro.

Paidós (Barcelona)

SCHANK, R. C. Y ABELSON, R. P.: Guiones, planes, metas y entendimiento.

Promociones y Publicaciones Universitarias (Barcelona)

GROS, B.: Aprender mediante el ordenador.

Tecnos (Madrid)

CORRAliza, J. A.: la experiencia del ambiente. Percepción y significado del medio construido.

Universidad Autónoma de Madrid (Madrid)

JIMÉNEZ, A.: Marcadores emocionales de la conducta.

Visor/Aprendizaje - Ministerio de Educación y Ciencia (Madrid)

AIVArez, A. (Comp.): Psicología y educación. Realizaciones y tendencias actuales en la investigación y en la präctica.

LEVINE, R. A., y WHITE, M. I.: El becho bumano. Las bases culturales del desarrollo educativo.

WALLON. H. (Comp. J. Palacios): Psicología y educación del niño.

Visor/Aprendizaje

TOESCA, Y.: El niño de 2 a 10 años (2.. edición).

Fe de errores

En la página 48 del número 39-40 de Infancia y Aprendizaje (artículo de Juan A. Llorens) donde dice aen el que se contemplan...» debe decir «en el que no se contemplan...». 